\title{
Narration de la subjectivité ou Comment s'atteindre
}

\author{
Renée-Berthe Drapeau
}

\section{The Narration of subjectivity or How to catch up to myself}

\begin{abstract}
Poised, pencil in hand, to write, (Drapeau) feels uncertain, released by the battles of those before her, but immobilised by her past. Writing implies an elongation of being. The problematic of subjectivity weaves its way through Drapeau's book, N'entendre qu'un son, in which fragments of narrative reveal the female character, designated by the pronoun 'you' (second person plural, vous) to the reader and to the female character herself. The 'you' is a melange of ' $I$ ' and 'she', and her identity remains fluid and permits the writer to identify with all her readers. The discouraging voices of the past and the relation to the maternal figure must be battled through the text, which shows that for certain women the battle is still to be fought. The young writer searches for a territory which she does not find; subjectivity still eludes her.
\end{abstract}

\section{L'héritage}

Certes de mot 'femme' ne semble plus posséder les vertus commerciales qu'il a eues; la mode est passée et la jeune écrivaine, le crayon suspendu auxdoigts, retrouve une place floue, indéfinie, celle qu'ellea toujours occupée malgré les apparences. Car même si la société s'est ouverte à sa parole, permissivité mouvante obtenue par des combats auxquels elle a aussi participé, elle conserve le mauvais héritage d'un surmoi griffu. Des choreutes murmurentà son oreille que créér nesert à rien, que c'est l'affaire de Dieu seul. Pour qui se prend-elle, cette auteure née en 1959, année de la mort de Duplessis? Souvent elle croit $n^{\prime}$ être personne tant sa famille la rive au passé et l'y immobilise. Elle garde parfois une attitude crispée et, s'observant comme elle visionnerait un film, elle ne se reconnaît pas dans cette fille qui recule, qui a toujours un peu peur, un peu mal.

Elle n'aime pas ce carcan qui la retient par en dedans. Autour d'elle, 
elle le remarque bien, toutes portent un peu les mêmes marques du corset social trop fraîchement enlevé. Les modèles qui la font écrire, et qu'elle nomme ses aînées, ou encore ses héroïnes, ont dû arracher de leur peau l'objet qui arrondit le dos et coupe la respiration. La résistance des vieux moules exige la constance du travail, son recommencement. L'écrivaine de trente ans que je suis n'échappe pas aux nécessités: ou bien elles'évalue d'un oeil myope ou bien elle fonce vers la liberté et les risques qui l'effraient. Ecrire implique donc pour moi une activité d'élongation de l'être, comme dans l'antigymnastique de Thérèse Bertherat, qui libère les muscles, les assouplit et allonge la colonne vertébrale en la dépliant. Dans ma pratique littéraire je reprends des couloirs empruntés par mes aînées, comme elles je couche sur des balles et des bâtons ${ }^{1}$ les parties nouées de mon image intérieure. J'écris la plongée vers des douleurs qui nous sont communes. J'opère sur un corps tissu de mots, pas toujours les bons, qu'il faut reformuler afin que se déplie une identité parcourue de racines vigoureuses.

\section{$N^{\prime}$ entendre qu'un son et la voix}

Dans les livres de femmes, le je ne va pas de soi, il se fragmente et mille fois perce la même coquille d'oeuf, geste renouvelé de la naissance à soi-même. Ce mouvement éternellement semblable est une construction; chaque brisure de l'oeuf contribue à l'édification d'une autonomie, d'une confiance en sa voix. La question de la subjectivité traverse tous mes textes, en particulier le roman $N^{\prime}$ entendre qu'un son. J'y interroge les rapports familiaux et la relationà la mère. Mon héroïne ne sait qui elle est, elle rêve qu'elle perd ses dents; elle est assaillie de toutes parts et ne songe qu'à prendre la fuite. En même temps, elle cherche ses origines et fait un genre d'enquête pour s'assurer de la véracité de ses souvenirs d'enfance, de sa pensée. Son être n'est pas unifié, mais il ne saurait l'être, elle perdrait son temps à ne parler qu'une langue et à ne fréquenter qu'une culture-à s'enfermer dans de nouveaux ou de vieux stéréotypes. Le multiple lui appartient, il lui faut surtout y mettre de l'ordre en reconnaissant le rôle de sa famille dans sa vie. Le texte ressemble donc à ces albums aux photos parfois floues, parfois claires. Des fragments de récit révèlent petit à petit aux lecteurs et à elle-même ce personnage féminin que je désigne par le pronom vous. 
Cette femme ne se reconnaît pas dans la société qui l'a formée. Ni la ville où elle vit (Montréal) ni sa province ne sont nommées, elle demeure une sorte d'étrangère et sa langue maternelle ne lui sert pas d'ancrage, puisqu'elle choisit d'écrire son roman en anglais pour que les espions de la famille, qui habitent toujours son esprit, ne viennent pas fouiner dans son texte. Aussi, elle ne peut être personne. Elle est n'importe qui et ne peut accéder à un je que par son nom d'auteure inscrit sur un manuscrit achevé, donc prêt à être LU. Autrement, c'est le travail sur les textes des autres (la traduction souvent publicitaire) et la jalousieà l'égard de celles qui osent, en l'occurence Virginia Woolf.

L'emploi du pronom aurait posé problème si la deuxième personne ne m'était pas venue tout de suite. J'aurais dû sans doute utiliser elle, qui m'aurait demandé quelque part l'usage d'un prénom au moins. Vous permet une identification de l'écrivaine tourmentée avec toute personne qui ouvre le roman pour le lire: 'Vous pourriez écrire ce roman dans la langue anglaise ...' Ce pronom lu à haute voix cependant se détache du lecteur, de la lectrice, pour appeler quelqu'un d'autre. Il tient de la correspondance. J'ai écrit $N^{\prime} e n t e n d r e ~ q u ' u n$ son comme si j'écrivais une lettre à quelqu'un qui serait ce personnage et dont les réponses consisteraient en ces petits plans de travail que je construisais à mesure. L'interlocutrice imaginaire, qui n'était que moi-même en acte d'écrire, me poussait à poursuivre ce roman, à le reprendre à chaque fois qu'un contrat de travail m'avait forcée à le laisser. Mais, dans les faits, quand on lit le roman, jedoute fort quecette idée de la correspondance nous vienne à l'esprit. La deuxième personne du pluriel résulte d'un mélange du je et du elle, et l'identité du personnage reste floue. L'intrigue réside en partie dans ce flou, cette incertitude d'où surgit le questionnement.

\section{La lunette embrouillée}

Ainsi, la narratrice qui organise le trajet du texte ne peut distribuerson savoir que par fragments. Parce que ne lui sont accessibles que des morceaux de vérité ou de fantasme. Tout entière prise dans l'univers de l'héroïne, elle s'enroule dans la deuxième personne du pluriel; sa vision se rétrécit: l'omniscience des romans classiques ne la servirait pas, les choses lui viennentautrement. Cette narratricese trompedonc dans ses interprétations et les corrigeensuite, ou bien elle neles corrige pas. Elle porte le discours de l'imagination et, s'il n'y a pas de chien au 
corps circulaire dans le métro, l'animal existe tout de même parce que la panne permet qu'il surgisse. Une série de tableaux se succèdent, indiquant les aspects du monde intérieur, l'angoisse de celle qui nesait pas d'où elle vient, qui elle est. C'est à mesure que le dialogue avec Elisabeth, sa mère, devient possible, que le sense rétrécit. La lecture ne livre que la vision tronquée de la romancière en quête de racines. Le rêve, les paysages de l'inconscient s'étalent. La voix narrative tient de la chorale et les renseignements donnés forment des noeuds pluriels.

Dans $N^{\prime} e n t e n d r e q u^{\prime} u n$ son, la communication est difficile. Chacun demeure muré dans son univers et n'écoute autrui que pour entendre sa propre histoire. Georges seul échappe à cette règle, mais son pouvoir n'est que celui des êtres humains, il ne peut lutter contre les monstres qui assaillent son amante. Il assiste impuissant à une destruction quis' opère hors delui. L'imaginairelui fend la bouche et il ne peut agir que minimalement. L'amie qu'il aime cherche partout des moyens de fuir la folie et plus elle fuit, plus elle s'enfonce. Monsieur Clébert traîne avec elle quelque cadavre et Jérôme s'entoure d'une buée de musique. Quant aux aînés de la famille, ils ne savent qu'interdire, ridiculiser. Ils donnent un sens bizarre à des comportements d'enfants et, en effet, ceux-ci deviennent étranges. Cernés d'ombre. Les sujets se détachent de leur jardin de naissance et ils doivent se trouver une terre: New York, Paris ..., des villes de roman. Le frère, lui, s'en tire mieux, le sol ne s'est pas tout à fait dérobé sous ses pieds. Il se trouve une langue muette et bavarde, la grandelangue émouvante du saxophone. Pendant qu'il crée, sa soeur essaie d'écrire elle aussi dans l'ailleurs de la langue maternelle.

Son itinéraire à elle, qui jouait du piano étant petite, imite les sons brisés de la musique contemporaine. La narration prend l'allure des symphonies fragmentaires issues des bruits du monde concret et de leur transformation sur bandes. Il s'agit de nommer le f(l)ou, les grumeaux qui échappent à la maîtrise. Accueillir l'indicible pour que sa force ne détruise plus l'être et qu'il devienne plutôt source d'invention. Le lapsus est vert dans ce roman, il contient une part de printemps inaccessible à la sujette qui s'arc-boute. Il faut que la créatrice d'Anne Vaucaire affronte le passé qui l'obsède sous la forme de klaxons, 'she has to face the music'. 'Vous ne voulez pas écrire vos histoires de famille', écris-je. Cette famille est une somme de discours, 'just a pack of cards'; il s'agit de retrouver son langage propre. 


\section{Territoires}

Cette quête concerne le territoire perdu, une langue voléeà reprendre. Dépourvue de lieu au sein du monde maternel, trop vite chassée de celui-ci, mon héroïne ne peut pas avoir accès à elle-même. Pour écrire, elle utilise des trucs, mais elle ne finit pas ses livres. The Wait ne s'achève qu'une fois le territoire franchement revendiqué et la culpabilité chassée, c'est-à-dire vers la fin du roman. La libération se fait contre une matière qui rend les chairs ratatinées. Elle n'existe que lorsque le sentiment d'avoir tort s'éloigne. En écrivant $N^{\prime} e n t e n d r e$ $q u$ 'un son, j'ai sans cesse été préoccupée par ce thème de la culpabilité qui arrête le désir et qui tue le sujet à petit feu. Le combat du personnage principal du texte, et le mien, se fait contre des voix décourageantes. Des paroles de juges qui enferment. Ces voix du passé demeurent toujours présentes dans l'imaginaire et des mots simples comme côte en anglais ne viennent pas s'inscrire sur la feuille. Les exercices ordinaires deviennent ardus comme des exploits.

En apparence, la traductrice évite le carcan traditionnel, elle travaille et fréquente des hommes sans grand souci des convenances. Le corset ne retient toujours un morceau de son cerveau, elle n'a pas confiance en elle-même et son énergie créatrice est brûlée par la peur. La fonction sociale de la narration passe par cette révélation des craintes toujours vives. $N^{\prime}$ entendre $q u^{\prime} u n$ son $n^{\prime}$ est pas un manifeste, mais il montre que la douleur existe et que pour certaines femmes tout $n$ 'est pas gagné. De vieilles attitudes de repli guettent celles qui nes'accueillent pas et qui ne peuvent jouer pour elles-mêmes le rôle de la bonne mère. Outre Elisabeth, les femmes n'existent pas tellement dans la vie de mon héroïne; exilée des sources nourricières, elle se perd, elle cherche et ne peut plus que pleurersur un grand vide.Comme Clébert, qui entretient la voix de son épouse décédée, elle ne possède qu'un cadavre de femme, lui-même associé au cadavre imaginédu père que renverse un camion. Georges soutient, il materne, mais il nedonne pas $d^{\prime}$ identité à qui croit n'en pas avoir. Il est l'autre du couple idéal et un morceau manque du côté féminin.

Ce manque conduit à une petite névrose dite portative. La traductrice ne devient pas un de ces fantômes qui hantent les couloirs des cliniques. Elle porte lemal de vivre des gens ordinaires quevivent sous une cloche de détresse. Cependant autour existent des outils de libération: de multiples langues, des films, de la musique audacieuse ... Des 
perches se tendent. Elisabeth et sa fille vont au cinéma, elles s'affrontent après un concert, elles s'écrivent. Le son unique se multiplie et la parole peut enfin surgir. Dans l'appartement de Jérôme, une discussion difficile est entreprise et on se doute que ce grand nettoyage des affects donnera naissance à des êtres neufs. La réconciliation mène au partage d'abord impossible des territoires.

La figure maternelle revient souvent dans mes textes, car je crois que la relation avec celle qui nous a mise au monde garde généralement beaucoup d'ambivalence. L'image qu'on se fait d'elle nous forme et nous déforme. Il est question de s'approprier du féminin, de trouver sa place dans une filiation et devenir finalement adulte: une autre vis-à-vis d'ELLE. La différence que permet la signature n'advient pas encore dans $N^{\prime}$ entendre qu'un son, mais elle apparaît de manière symbolique à travers les chants d'Oum Koulsoum et les références à l'étranger. Elle est évoquée dans la multiplicité des lieux. La jeune écrivaine se cherche un territoire et ne le trouve pas; tel un oiseau migrateur, elle se déplace, éternelle étrangère exilée d'elle-même. Le multiculturalisme ne parle que du désir non réalisé. Le désir d'être autre pour l'autre, c'est-à-dire d'être soi et d'en jouir.

\section{Notes}

1. Quelques outils utilisés en gymnastique dite douce. 